\title{
Outcomes of Stenotrophomonas maltophilia hospital-acquired pneumonia in intensive care unit: a nationwide retrospective study
}

Philippe Guerci ${ }^{1}, 2$, Hugo Bellut ${ }^{3}$, Mokhtar Mokhtari ${ }^{1}$, Julie Gaudefroy ${ }^{4}$, Nicolas Mongardon ${ }^{5}$, Claire Charpentier ${ }^{6}$, Guillaume Louis ${ }^{7}$, Parvine Tashk ${ }^{8}$, Clément Dubost ${ }^{9}$, Stanislas Ledochowski ${ }^{10}$, Antoine Kimmoun ${ }^{11}$, Thomas Godet ${ }^{12}$, Julien Pottecher ${ }^{4,13}$, Jean-Marc Lalot ${ }^{14}$, Emmanuel Novy ${ }^{1}$, David Hajage ${ }^{15}$, Adrien Bouglée $3^{*}$ (D) and AZUREA research network

\begin{abstract}
Background: There is little descriptive data on Stenotrophomonas maltophilia hospital-acquired pneumonia (HAP) in critically ill patients. The optimal modalities of antimicrobial therapy remain to be determined. Our objective was to describe the epidemiology and prognostic factors associated with S. maltophilia pneumonia, focusing on antimicrobial therapy.

Methods: This nationwide retrospective study included all patients admitted to 25 French mixed intensive care units between 2012 and 2017 with hospital-acquired S. maltophilia HAP during intensive care unit stay. Primary endpoint was time to in-hospital death. Secondary endpoints included microbiologic effectiveness and antimicrobial therapeutic modalities such as delay to appropriate antimicrobial treatment, mono versus combination therapy, and duration of antimicrobial therapy.
\end{abstract}

Results: Of the 282 patients included, 84\% were intubated at S. maltophilia HAP diagnosis for duration of 11 [5-18] days. The Simplified Acute Physiology Score II was 47 [36-63], and the in-hospital mortality was 49.7\%. Underlying chronic pulmonary comorbidities were present in $14.1 \%$ of cases. Empirical antimicrobial therapy was considered effective on $S$. maltophilia according to susceptibility patterns in only $30 \%$ of cases. Delay to appropriate antimicrobial treatment had, however, no significant impact on the primary endpoint. Survival analysis did not show any benefit from combination antimicrobial therapy $(\mathrm{HR}=1.27,95 \% \mathrm{Cl}[0.88 ; 1.83], p=0.20)$ or prolonged antimicrobial therapy for more than 7 days $(\mathrm{HR}=1.06,95 \% \mathrm{Cl}[0.6 ; 1.86], p=0.84)$. No differences were noted in in-hospital death irrespective of an appropriate and timely empiric antimicrobial therapy between mono- versus polymicrobial S. maltophilia $\operatorname{HAP}(p=0.273)$. The duration of ventilation prior to $S$. maltophilia HAP diagnosis and ICU length of stay were shorter in patients with monomicrobial $S$. maltophilia HAP ( $p=0.031$ and $p=0.034$ respectively).

Conclusions: S. maltophilia HAP occurred in severe, long-stay intensive care patients who mainly required prolonged invasive ventilation. Empirical antimicrobial therapy was barely effective while antimicrobial treatment modalities had no significant impact on hospital survival.

Trial registration: clinicaltrials.gov, NCT03506191

Keywords: Hospital-acquired pneumonia, Stenotrophomonas maltophilia, Intensive care, Antimicrobial therapy, Combination therapy

\footnotetext{
* Correspondence: adrien.bougle@aphp.fr

${ }^{3}$ Sorbonne Université, Assistance Publique - Hôpitaux de Paris (AP-HP),

Department of Anaesthesiology and Critical Care Medicine, Institute of

Cardiology, Pitié-Salpêtrière Hospital, 47-83 Boulevard de I'Hôpital, 75013

Paris, France

Full list of author information is available at the end of the article
}

(c) The Author(s). 2019 Open Access This article is distributed under the terms of the Creative Commons Attribution 4.0 International License (http://creativecommons.org/licenses/by/4.0/), which permits unrestricted use, distribution, and reproduction in any medium, provided you give appropriate credit to the original author(s) and the source, provide a link to the Creative Commons license, and indicate if changes were made. The Creative Commons Public Domain Dedication waiver (http://creativecommons.org/publicdomain/zero/1.0/) applies to the data made available in this article, unless otherwise stated. 


\section{Background}

Stenotrophomonas maltophilia is one of the 10 most frequently isolated pathogens responsible for hospitalacquired pneumonias (HAPs) in intensive care unit (ICU) patients in western countries $[1,2]$, representing approximately $5 \%$ of positive pulmonary samples. Previous studies identified several risk factors for developing S. maltophilia HAP in critically ill patients, such as prolonged ICU hospitalization associated with invasive procedures, extended periods of mechanical ventilation, or exposure to broad-spectrum antibiotics [3-5]. Therefore, $S$. maltophilia pneumonia occurs preferentially in patients with the poorest prognosis [6, 7]. However, these studies were conducted from heterogeneous and small cohorts of patients. The severity of S. maltophilia HAP and antimicrobial therapy modalities were sparsely reported $[3,4]$. Hence, data are lacking to draw recommendations on the optimal therapeutic strategies against S. maltophilia pneumonia.

We undertook a large nationwide multicenter retrospective study with the main objective to demonstrate that modalities of antibiotic therapy, including empirical antimicrobial choice, whether a combination therapy was used, or the duration of the therapy, would influence in-hospital mortality. Secondary objectives were to describe the characteristics of ICU patients with S. maltophilia HAP and to draw prognostic factors of these pneumonias.

\section{Methods}

\section{Design of the study and setting}

The medical records of patients who experienced S. maltophilia pneumonia from January 2012 to January 2017 were collected from 25 ICUs of the French Society of Anaesthesia \& Intensive Care Medicine (SFAR) and AZUREA networks [8]. Participating centers and casemixes are listed in Additional file 1.

The collected data involved both ICU and hospital stays. Follow-up was stopped either after hospital discharge or death, whichever occurred first.

\section{Participants \\ Eligibility criteria}

All patients aged over 18 years who were admitted to the participating ICUs and presenting with a documented diagnosis of S. maltophilia pneumonia during their ICU stay were eligible.

\section{Source and method of selection}

The patient's files were extracted through French hospital discharge database containing individual records of all hospital stays using International Classification of Disease (ICD-10) for the terms "Stenotrophomonas maltophilia" and "pneumonia." In addition, ICU medical charts were cross-checked with microbiology laboratoryspecific information systems to ensure exhaustivity.

Each medical record was analyzed by local investigators to determine if clinical, biological, and/or radiological signs of $S$. maltophilia HAP were present, thus excluding respiratory tract colonizations (defined as a positive respiratory sample without clinical, biological, and/or radiological signs of $S$. maltophilia pneumonia). In case of uncertainty, consensus was obtained between local infectious disease specialists and study coordinators (PG, AB) to clarify S. maltophilia HAP cases.

\section{Definitions}

Pneumonia was defined as follows: (i) new or progressive lung infiltrate, (ii) temperature $>38^{\circ} \mathrm{C}$ or $<36.5^{\circ} \mathrm{C}$, leukocyte count $>12,000 \mu \mathrm{l}^{-1}$ or $<4000 \mathrm{\mu l}^{-1}$, purulent endotracheal aspirate or sputum, (iii) positive respiratory sample (see below), and (iv) decline in oxygenation [9, 10]. HAP was defined as a pneumonia not incubating at the time of hospital admission and occurring $48 \mathrm{~h}$ or more after admission. Ventilator-associated pneumonia (VAP) was defined as a pneumonia occurring $48 \mathrm{~h}$ or more after tracheal intubation [9].

The clinical cure of S. maltophilia pneumonia was defined by the absence of pneumonia criteria $48 \mathrm{~h}$ after antimicrobial therapy cessation. Treatment failure was defined as a failure of first-line treatment or death attributable to $S$. maltophilia pneumonia. Recurrence was defined as the onset of new pneumonia criteria associated with a positive respiratory sample with $S$. maltophilia after the initial pneumonia was considered successfully cured.

Empirical antimicrobial therapy was defined as the first agents prescribed for the initial treatment of HAP (effective or not on $S$. maltophilia) finally diagnosed as being caused by $S$. maltophilia. Empirical antimicrobial therapy was considered as effective if the S. maltophilia strain cultured from the respiratory sample was susceptible to at least one of the antimicrobial agents. Combination therapy was defined as the administration of at least two antimicrobial agents a priori (before $S$. maltophilia HAP has been confirmed, usually within $48 \mathrm{~h}$ ) or a posteriori (after S. maltophilia HAP has been confirmed) effective on the S. maltophilia strain for more than $24 \mathrm{~h}$.

\section{Data collection}

Usual demographic variables were collected, including previous hospital stays and previous exposure to antimicrobial therapies (agents and durations). Simplified Acute Physiology Score II (SAPSII) and the Sequential Organ Failure Assessment (SOFA) score were assessed.

On the day of $S$. maltophilia HAP diagnosis, the SOFA score was collected, as well as the number and type of 
invasive devices inserted. The severity of hypoxemia was graded according to the Berlin acute respiratory distress syndrome (ARDS) criteria [11]. Requirements for highflow nasal oxygen therapy, non-invasive or invasive mechanical ventilation, or extracorporeal membrane oxygenation (ECMO) were reported. Empirical antimicrobial therapy and secondary adaptations were recorded, as were durations.

\section{Diagnosis of positive bacterial culture}

In case of $S$. maltophilia isolation, the culture was considered to be positive (either mono- or polymicrobial infection) with the following cutoff: (1) for minimally contaminated lower respiratory tract sample with quantitative culture, the threshold was $10^{4}$ colony-forming units $(\mathrm{CFU}) / \mathrm{ml}$ for bronchoalveolar lavage (BAL) and the cutoff was $10^{3} \mathrm{CFU} / \mathrm{ml}$ for protected specimen brush (PSB) or protected (plugged) telescoping catheter (PTC); (2) nonprotected sample (endotracheal aspirate, ETA) with quantitative culture $\left(10^{5} \mathrm{CFU} / \mathrm{ml}\right)$; or (3) sputum bacteriology with quantitative culture $\left(10^{7} \mathrm{CFU} / \mathrm{ml}\right)$ [12].

\section{Antimicrobial susceptibility testing (AST)}

S. maltophilia identification characteristics (date of isolation and type of respiratory tract sampling) and antimicrobial susceptibility testing were independently performed by each microbiology laboratory. AST was performed on isolates using disk diffusion or automated testing methods according to guidelines and breakpoints established by the European Committee on Antimicrobial Susceptibility Testing (EUCAST) [13].

\section{Data management}

Data were collected and managed using Research Electronic Data Capture (REDCap) software [14]. The database was approved by the institutional review board of the SFAR (IRB00010254-2015-010), which waived the need for signed informed consent of the participants, in accordance with the French legislation on noninterventional studies [15]. The study was declared on clinicaltrials.gov (NCT03506191). This manuscript was written in accordance with the STROBE statement for the reporting of observational studies in epidemiology.

\section{Statistical analysis}

The results are expressed as the number of patients (\%) for categorical variables and mean ( \pm standard deviation) or median [IQR] for continuous variables.

Prognostic factors associated with time to in-hospital death were studied using the Cox proportional hazard model. Time to in-hospital death was calculated from the diagnostic date of $S$. maltophilia to death. The follow-up was censored at discharge from the ICU and/ or the hospital. Baseline prognostic factors were age,
SAPS II, mechanical ventilation at diagnosis, VAP, duration of mechanical ventilation before the diagnosis, SOFA score at diagnosis, bacteremia, mono/polymicrobial pneumonia, use of empirical antimicrobial therapy, and use of empirical antimicrobial therapy effective against S. maltophilia. Other antimicrobial therapyrelated variables were not defined as baseline and were thus entered in the model as time-dependent variables, including time elapsed between sample and effective antimicrobial therapy, use of effective combination antimicrobial therapy, and duration of effective antimicrobial therapy against S. maltophilia (monotherapy or combination therapy).

Baseline and time-dependent variables associated $(p<$ 0.05 ) with outcome in the univariate analysis and that were present at the diagnosis were considered for the multivariate model, and the final model was selected using backward stepwise regression $(p<0.05)$. Hazard ratios (HR) were calculated accordingly with their 95\% confidence intervals (CI).

We compared the time to in-hospital death between patients who received or not an empirical antimicrobial therapy effective against $S$. maltophilia using propensity score framework. The variables used for propensity score estimations were age, sex, SOFA score at diagnosis, SAPS II, and the ICU length of stay before pneumonia diagnosis. The two groups of patients were matched using a 1:1 nearest neighbor matching algorithm with replacement, using a caliper of 0.2 of the standard deviation of the propensity score on the logit scale [16]. Covariate balance between the two groups was assessed after matching, and we considered an absolute standardized difference (ASD) less than 0.1 as evidence of balance [17]. Then, time to in-hospital death was compared between matched groups using a Cox proportional hazard model. The 95\% confidence intervals of the estimated hazard ratio (empirical antimicrobial therapy yes vs no) were estimated using robust standard error [18].

Significance was defined as $p$ values $<0.05$. Statistical tests were two-sided. Statistical analyses were performed using R 3.5.0 (R Foundation for Statistical Computing, http://www.R-project.org/, Vienna, Austria).

\section{Results \\ Population}

Of the 102,316 patients admitted to the 25 ICUs within the study period, $282(0.27 \%)$ with a $S$. maltophilia pneumonia were included (Fig. 1). Our population was predominantly male (69.9\%), with an age of 65 [56-74] years, mostly admitted for medical reasons $(59.2 \%)$ or emergent surgery (29.4\%). Severity at admission was illustrated by SOFA score (8 [5-11]) and SAPSII (47 [36$63])$. ICU and hospital lengths of stay were 32 [19-58] and 54 [30-94] days, respectively. The overall in- 


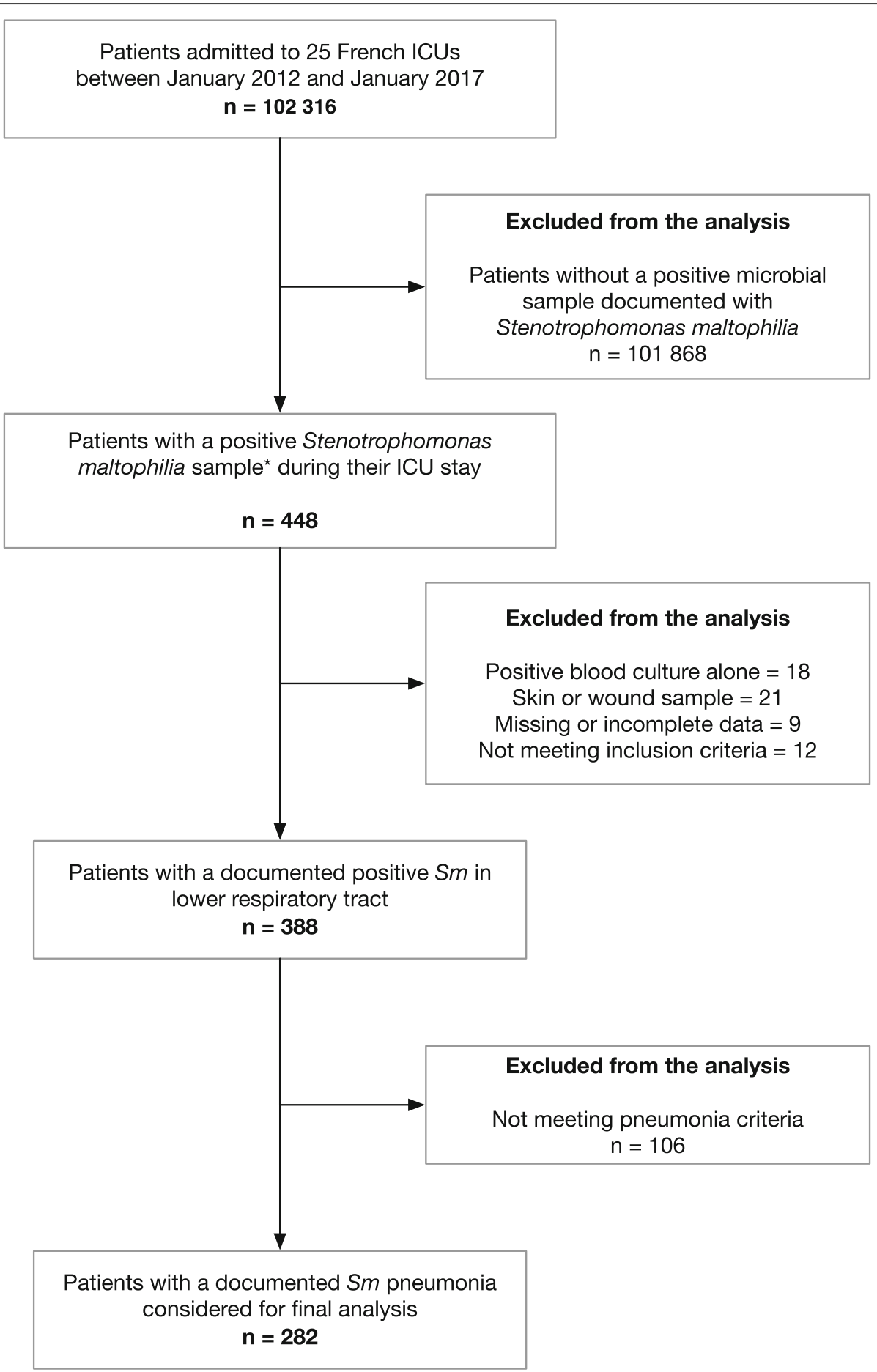

Fig. 1 Flowchart of the inclusion of patients presenting with Stenotrophomonas maltophilia hospital-acquired pneumonia. Asterisk indicates that sample can be from lower respiratory tract, blood, wound/skin, or urine

hospital mortality rate was $49.7 \%$ (Table 1 ). There was no difference in trends of patient inclusion and distribution over years. $48,51,60,65$, and 59 patients were included in 2012, 2013, 2014, 2015, and 2016 respectively with similar distributions in terms of mortality.
Patients had been hospitalized in the ICU for 11 [5-19] days at the time of onset of S. maltophilia pneumonia. Forty patients (14.2\%) presented with a chronic underlying pulmonary disease. Other characteristics of patients are described in Table 1 , and invasive 
Table 1 Demographic and baseline characteristics of ICU patients with Stenotrophomonas maltophilia hospital-acquired pneumonia

\begin{tabular}{|c|c|}
\hline Variables & Total $N=28$ \\
\hline Gender, male & $197(69.9)$ \\
\hline Age, years & $65[56-74]$ \\
\hline BMI $\left(\mathrm{kg} \mathrm{m}^{-2}\right)$ & $25.3[22-28$. \\
\hline \multicolumn{2}{|l|}{ Reason for ICU admission } \\
\hline Medical condition & $167(59.2)$ \\
\hline Scheduled surgery & $32(11.3)$ \\
\hline Emergent surgery & $83(29.4)$ \\
\hline $\begin{array}{l}\text { Postoperative period of } \\
\text { cardiac surgery }\end{array}$ & $60(21.3)$ \\
\hline Previous carriage of S. maltophilia & $13(4.6)$ \\
\hline \multicolumn{2}{|l|}{ Pulmonary comorbidities } \\
\hline $\begin{array}{l}\text { Chronic obstructive pulmonary } \\
\text { disease }\end{array}$ & $51(18.1)$ \\
\hline Chronic respiratory insufficiency & $30(10.6)$ \\
\hline Cystic fibrosis & $2(0.7)$ \\
\hline \multicolumn{2}{|l|}{ Other comorbidities } \\
\hline Hypertension & $147(52.1)$ \\
\hline Congestive heart failure & $66(23.4)$ \\
\hline $\begin{array}{l}\text { Dialysis-dependent chronic } \\
\text { kidney disease }\end{array}$ & $8(2.8)$ \\
\hline Liver cirrhosis & $22(7.8)$ \\
\hline Insulin-requiring diabetes & $31(11)$ \\
\hline Severe neurologic disability & $30(10.6)$ \\
\hline \multicolumn{2}{|l|}{ Habits } \\
\hline Active smoking & $86(30.5)$ \\
\hline Chronic alcohol abuse & $47(16.7)$ \\
\hline Drug abuse & $8(2.8)$ \\
\hline \multicolumn{2}{|l|}{ Immune comprise conditions ( $n, \%)$} \\
\hline Neutropenia & $1(0.3)$ \\
\hline HIV and/or CD4 count $<50 / \mathrm{mm}^{3}$ & $1(0.3)$ \\
\hline Recent or ongoing chemotherapy & $11(3.9)$ \\
\hline Hematologic malignancy & $16(5.7)$ \\
\hline Solid tumor being actively treated & $20(7.1)$ \\
\hline Solid tumor in remission & $22(7.8)$ \\
\hline $\begin{array}{l}\text { Immunosuppressive therapy or } \\
\text { corticosteroids use }>0.5 \mathrm{mg} / \mathrm{kg} \\
>30 \text { days }\end{array}$ & $34(12)$ \\
\hline $\begin{array}{l}\text { Innate or acquired immune } \\
\text { deficiency }\end{array}$ & $1(0.3)$ \\
\hline \multicolumn{2}{|l|}{ Severity scores } \\
\hline SOFA score at admission & $8[5-11]$ \\
\hline SAPS $\|$ at $24 \mathrm{~h}$ & $47[36-63]$ \\
\hline Ventilator-associated pneumonia & $228(80.8)$ \\
\hline $\begin{array}{l}\text { Duration of ventilation before } S \text {. } \\
\text { maltophilia HAP days }\end{array}$ & $11[5-18]$ \\
\hline
\end{tabular}

Table 1 Demographic and baseline characteristics of ICU patients with Stenotrophomonas maltophilia hospital-acquired pneumonia (Continued)

\begin{tabular}{ll}
\hline Variables & Total N=282 \\
\hline ICU length of stay, days & $32[19-58]$ \\
Hospital length of stay, days & $54[30-94]$ \\
$\begin{array}{l}\text { Number of days between hospital } \\
\text { admission and S. maltophilia HAP }\end{array}$ & $16[8-27]$ \\
Number of days between ICU & $11[5-19]$ \\
admission and S. maltophilia HAP & \\
Mortality & $140(49.7)$ \\
Mortality directly attributable to & $34(24.3)$ \\
S. maltophilia &
\end{tabular}

Data are expressed as number and percentage or median [interquartile range] as appropriate

$B M I$ body mass index, HAP hospital-acquired pneumonia, HIV human immunodeficiency virus, ICU intensive care unit, IQR interquartile range, SAPS Simplified Acute Physiology Score, SD standard deviation, SOFA Sequential Organ Failure Assessment, S. maltophilia Stenotrophomonas maltophilia

devices are reported in Additional file 2: Table S1 in the online data supplement.

\section{Description of Stenotrophomonas maltophilia hospital- acquired pneumonia}

Characteristics of S. maltophilia HAP are described in Table 2. Briefly, $41.6 \%$ of S. maltophilia pneumonias were monomicrobial and $80.8 \%$ were VAP. Blood culture was concomitantly positive in only $7.1 \%$ of cases.

Microbiological diagnosis methods for isolation of $S$. maltophilia are presented in Additional file 3: Table S2 in the online data supplement. Patients with S. maltophilia VAP had a duration of mechanical ventilation before the onset of pneumonia of 11 [5-18] days. S. maltophilia pneumonia-related septic shock was present in 123 patients (43.6\%) within $48 \mathrm{~h}$ of S. maltophilia HAP (septic shock attributed to pneumonia by clinicians and without other identified cause in the post hoc analysis). Among these patients who developed septic shock, 38 (30.8\%) did not receive initial empirical antimicrobial therapy. Forty-nine percent of patients fulfilled moderate or severe ARDS criteria.

\section{Antimicrobial therapy}

The description of antimicrobial therapy modalities is reported in Table 3. Before the onset of S. maltophilia pneumonia, patients received 3 [2-4] prior antimicrobial therapies for at least 5 consecutive days in ICU. Empirical antimicrobial therapy was a posteriori effective against $S$. maltophilia in $30.1 \%$ of cases. The duration of effective antimicrobial therapy on S. maltophilia was 11 [7-15] days. A combination of antimicrobials effective against $S$. maltophilia was used in $59.4 \%$ of patients for 7 [5-12] days. 
Table 2 Characteristics of Stenotrophomonas maltophilia hospital-acquired pneumonia

\begin{tabular}{|c|c|}
\hline Variables & Total $N=282$ \\
\hline $\begin{array}{l}\text { Monomicrobial pneumonia (S. maltophilia only) } \\
(n,(\%))\end{array}$ & $117(41.6)$ \\
\hline \multicolumn{2}{|l|}{$\begin{array}{l}\text { Other microorganisms identified (polymicrobial) } \\
(n,(\%))\end{array}$} \\
\hline Acinetobacter baumanii & $7(4.3)$ \\
\hline Citrobacter spp. & $7(4.3)$ \\
\hline Enterobacter spp. & $30(18.3)$ \\
\hline Enterococcus spp. & $6(3.7)$ \\
\hline Escherichia coli & $25(15.2)$ \\
\hline Haemophilus & $3(1.8)$ \\
\hline Hafnia alvei & $3(1.8)$ \\
\hline Klebsiella spp. & $25(15.2)$ \\
\hline Methicillin-resistant Staphylococcus aureus & $3(1.8)$ \\
\hline Methicillin-sensitive Staphylococcus aureus & $13(7.9)$ \\
\hline Morganella morganii & $1(0.6)$ \\
\hline Proteus spp. & $7(4.3)$ \\
\hline Pseudomonas aeruginosa & $49(29.9)$ \\
\hline Serratia spp. & $8(4.9)$ \\
\hline Streptococcus spp. & $1(0.6)$ \\
\hline Others & $10(6.1)$ \\
\hline \multicolumn{2}{|l|}{ S. maltophilia pneumonia-related manifestations ( $n,(\%))$} \\
\hline Associated bacteremia & $20(7.1)$ \\
\hline Associated empyema thoracis & $25(8.9)$ \\
\hline Associated septic shock & $123(43.6)$ \\
\hline $\begin{array}{l}\text { Total number of days of norepinephrine infusion } \\
\text { (median [IQR]) }\end{array}$ & $0[0-6]$ \\
\hline \multicolumn{2}{|l|}{ Severity of pneumonia } \\
\hline \multicolumn{2}{|l|}{ Oxygenation level regarding S. maltophilia HAP $(n,(\%))$} \\
\hline $\begin{array}{l}\text { Hypoxemia with } \mathrm{PaO}_{2} / \mathrm{FIO}_{2}>300 \mathrm{mmHg} \text { with } \\
\text { PEEP } \geq 5 \mathrm{cmH}_{2} \mathrm{O}\end{array}$ & $45(16)$ \\
\hline Mild ARDS & $98(34.7)$ \\
\hline Moderate ARDS & $101(35.8)$ \\
\hline Severe ARDS & $36(12.7)$ \\
\hline Prone positioning $(n,(\%))$ & $13(4.6)$ \\
\hline ECMO requirement & $15(5.3)$ \\
\hline Veno-venous & $8(53.3)$ \\
\hline Veno-arterial & $7(46.7)$ \\
\hline
\end{tabular}

Data is presented as number (\%) or median [IQR]. Mild ARDS (200 mmHg $<\mathrm{PaO}_{2} /$ $\mathrm{FiO}_{2} \leq 300 \mathrm{mmHg}$ with PEEP $\left.\geq 5 \mathrm{cmH}_{2} \mathrm{O}\right)$, moderate ARDS $\left(100 \mathrm{mmHg}<\mathrm{PaO}_{2} / \mathrm{FiO}_{2} \leq\right.$ $200 \mathrm{mmHg}$ with PEEP $\left.\geq 5 \mathrm{cmH}_{2} \mathrm{O}\right)$, severe ARDS $\left(\mathrm{PaO}_{2} / \mathrm{FiO}_{2} \leq 100 \mathrm{mmHg}\right.$ with PEEP $\geq 5 \mathrm{cmH}_{2} \mathrm{O}$ )

S. maltophilia, Stenotrophomonas maltophilia; ARDS, Acute Respiratory Distress Syndrome, was defined according to the Berlin definition; PEEP, positive end-expiratory pressure

\section{Microbiological data}

AST of S. maltophilia strains is presented in Fig. 2a. Trimethoprim-sulfamethoxazole (TMP-SMX) (88.1\%) and
Table 3 Antimicrobial therapy management related to Stenotrophomonas maltophilia hospital-acquired pneumonia

\begin{tabular}{ll}
\hline Variables & Total $N=282$ \\
\hline $\begin{array}{l}\text { Number of antimicrobial therapy lines administered } \\
\text { within } 30 \text { days before diagnosis (median [IQR]) }\end{array}$ & $3[2-4]$ \\
Number of days with initial ineffective antimicrobial & $2[2-3.5]$
\end{tabular}
therapy on S. maltophilia (median [IQR])

Most commonly prescribed antimicrobial agents before S. maltophilia HAP onset $(n,(\%))$

$\begin{array}{ll}\text { Amoxicillin } & 20(7.1) \\ \text { Amoxicillin-clavulanate } & 61(21.6) \\ \text { Third-generation cephalosporin } & 93(33) \\ \text { Cefepime } & 25(8.9) \\ \text { Ceftazidime } & 17(6) \\ \text { Ticarcillin } & 4(1.4) \\ \text { Ticarcillin-clavulanate } & 10(3.5) \\ \text { Piperacillin } & 15(5.3) \\ \text { Piperacillin-tazobactam } & 97(34.4) \\ \text { Carbapenem } & 63(22.3) \\ \text { Aminoglycoside } & 70(24.8) \\ \text { Fluoroquinolone } & 40(14.2) \\ \text { Trimethoprim-sulfamethoxazole } & 9(3.2) \\ \text { Glycopeptide } & 59(20.9) \\ \text { Metronidazole } & 32(11.3) \\ \text { Linezolid } & 31(11) \\ \text { Others } & 71(25.2) \\ \text { Empirical antimicrobial therapy (n, (\%)) } & 166(58.8) \\ \text { Number of antimicrobial agents for empirical } & 1[0-2] \\ \text { antimicrobial therapy (median, [IQR]) } & \\ \text { Efficient empirical therapy on S. maltophilia ( } \text {, (\%)) } & 50(30.1) \\ \text { Combination antimicrobial therapy (2 or more) } & 167(59.4) \\ \text { targeting S. maltophilia ( } n \text {, (\%)) } & 11[7-15] \\ \text { Duration of antimicrobial therapy targeting S. } & \\ \text { maltophilia, days (median, [IQR]) } & \\ \text { Duration of combined (2 or more) antimicrobial } \\ \text { therapy targeting S. maltophilia, days (median, [IQR]) } \\ \text { S. maltophilia is intrinsically resistant to amoxicillin, amoxicillin/clavulanate, } \\ \text { ticarcillin, piperacillin/tazobactam, carbapenems, and aminoglycosides } \\ \text { (EUCAST expert rules version 3.1-26 Sept 2016). Data are presented as } \\ \text { median, interquartile range ([IQR]), or number (percentage) (n, (\%)) } \\ \text { as appropriate } \\ \text { HAP hospital-acquired pneumonia } \\ \end{array}$

ticarcillin-clavulanate (73.3\%) remained highly active against more than two thirds of S. maltophilia strains. The main antimicrobial therapies prescribed to treat $S$. maltophilia HAP after identification were TMP-SMX (29\%), ciprofloxacin (25\%), and ticarcillin-clavulanate (24\%) (Fig. 2b).

\section{Prognosis}

Treatment failure occurred in 65 patients (23.1\%). Recurrence of $S$. maltophilia pneumonia was diagnosed in 


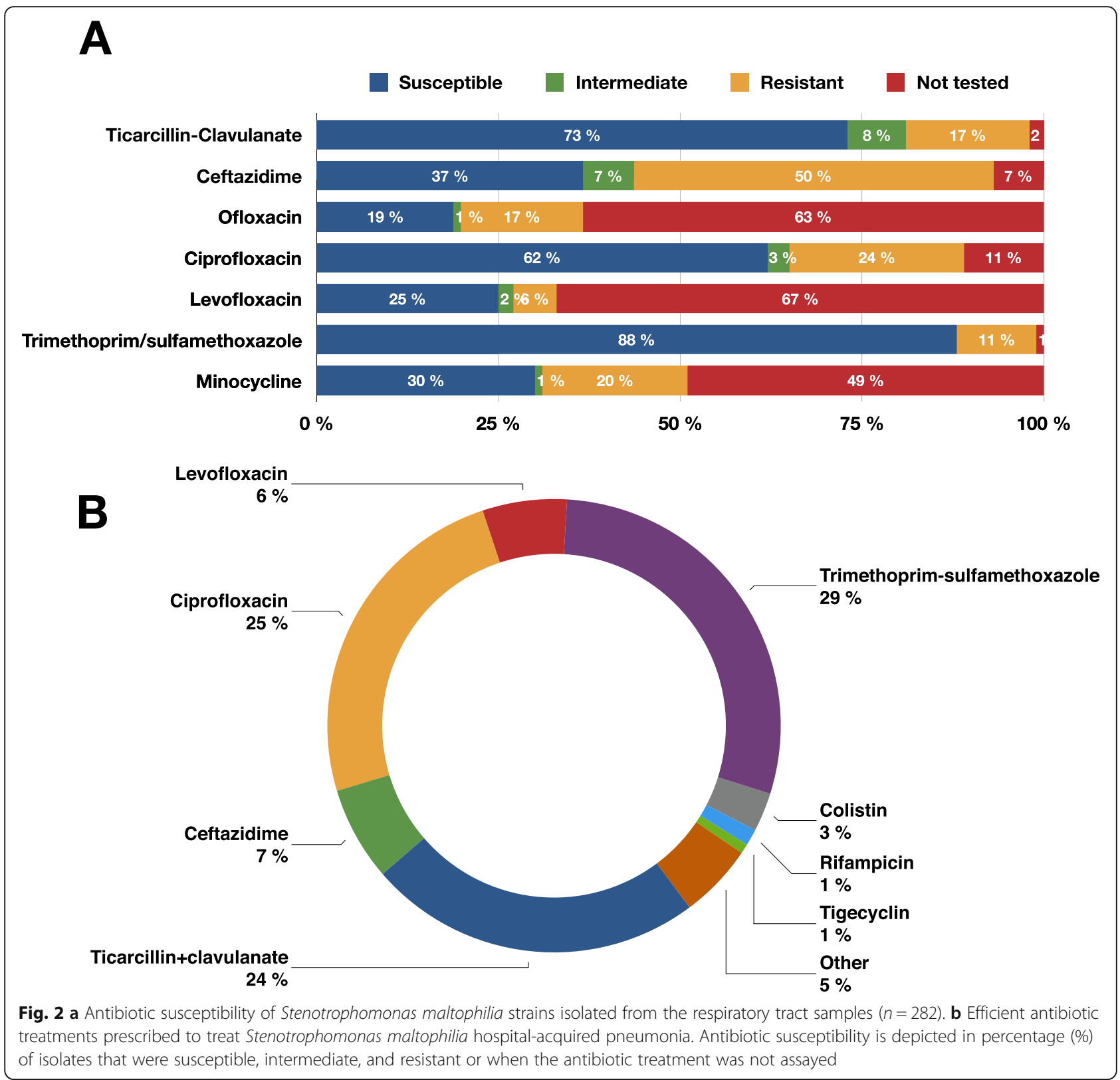

48 patients (17.0\%). Co-infection with another pathogen, mostly Pseudomonas aeruginosa $(n=30)$, was diagnosed in 70 patients $(24.8 \%)$ during recurrence (Additional file 4: Table S3).

In univariate analysis, variables associated with the primary endpoint (i.e., time to in-hospital death) were age $(\mathrm{HR}=1.025,95 \% \mathrm{CI}[1.012 ; 1.038], p=0.0001)$, SAPS II $(\mathrm{HR}=1.009$, 95\%CI $[1.001 ; 1.018], p=0.036)$, and SOFA score at pneumonia diagnosis $(\mathrm{HR}=1.099,95 \%$ CI $[1.057$; 1.142], $p<0.0001$ ) (Table 4). The subsequent occurrence of a septic shock was significantly associated with an increased risk of death $(\mathrm{HR}=3.070,95 \% \mathrm{CI}[1.9 ; 5.0], p<0.0001)$.
Neither the duration of treatment nor the use of combination therapy directed against $S$. maltophilia was associated with the primary endpoint (Table 4). Other commonly reported risk factors for S. maltophilia HAP (i.e., immunosuppression, chronic obstructive pulmonary disease (COPD), prior antimicrobial therapy) were not statistically associated with time to in-hospital death.

In multivariate analysis, only age $(\mathrm{HR}=1.02,95 \% \mathrm{CI}$ $[1.01 ; 1.04], p=0.001)$ and SOFA score at S. maltophilia pneumonia diagnosis $(\mathrm{HR}=1.1,95 \% \mathrm{CI}[1.06$; 1.15], $p<0.001)$ were associated with in-hospital death (Table 4). Subsequent septic shock was not included 
Table 4 Variables associated with the time to in-hospital death

\begin{tabular}{|c|c|c|c|c|}
\hline \multirow[t]{2}{*}{ Variables } & \multicolumn{2}{|l|}{ Univariate analysis } & \multicolumn{2}{|c|}{ Multivariate analysis } \\
\hline & $\mathrm{HR}[95 \% \mathrm{Cl}]$ & $\overline{p \text { value }}$ & $\mathrm{HR}[95 \% \mathrm{Cl}]$ & $p$ value \\
\hline Age & $1.025[1.012 ; 1.038]$ & 0.0001 & $1.02[1.01 ; 1.04]$ & 0.001 \\
\hline SAPS $\|$ & $1.009[1.001 ; 1.018]$ & 0.036 & & \\
\hline Mechanical ventilation at diagnosis & $1.692[0.83 ; 3.46]$ & 0.151 & & \\
\hline VAP & $0.748[0.49 ; 1.14]$ & 0.178 & & \\
\hline Duration of MV before the diagnosis & $0.997[0.988 ; 1.006]$ & 0.462 & & \\
\hline SOFA score at diagnosis & $1.099[1.057 ; 1.142]$ & $<0.0001$ & $1.1[1.06 ; 1.15]$ & $<0.001$ \\
\hline Bacteremia & $0.814[0.41 ; 1.60]$ & 0.551 & & \\
\hline Monomicrobial pneumonia & $1.368[0.98 ; 1.91]$ & 0.066 & & \\
\hline Co-infection with Pseudomonas aeruginosa & $0.843[0.53 ; 1.33]$ & 0.464 & & \\
\hline Time elapsed between pulmonary sample and effective antimicrobial therapy & $0.99[0.97 ; 1.01]$ & 0.434 & & \\
\hline$<24 h$ & 1 & & & \\
\hline $24-48 \mathrm{~h}$ & $1.22[0.74 ; 2]$ & 0.429 & & \\
\hline$>48 \mathrm{~h}$ & $1.34[0.85 ; 2.12]$ & 0.204 & & \\
\hline Empirical antibiotic therapy & $1.019[0.73 ; 1.43]$ & 0.914 & & \\
\hline Subsequent septic shock & $3.070[1.9 ; 5.0]$ & $<0.0001$ & & \\
\hline Empirical antibiotic therapy effective against S. maltophilia & $0.839[0.52 ; 1.35]$ & 0.4705 & & \\
\hline Effective combination antimicrobial therapy & $1.27[0.88 ; 1.83]$ & 0.204 & & \\
\hline Duration of effective antimicrobial therapy against S. maltophilia & $1.03[0.98 ; 1.07]$ & 0.243 & & \\
\hline$<7$ days & 1 & & & \\
\hline 7-14 days & $1.06[0.6 ; 1.86]$ & 0.842 & & \\
\hline$>14$ days & $0.88[0.45 ; 1.71]$ & 0.706 & & \\
\hline Duration of effective combination therapy against S. maltophilia & $0.99[0.96 ; 1.03]$ & 0.679 & & \\
\hline$<7$ days & 1 & & & \\
\hline 7-14 days & $0.82[0.52 ; 1.29]$ & 0.393 & & \\
\hline$>14$ days & $0.71[0.39 ; 1.29]$ & 0.262 & & \\
\hline
\end{tabular}

SAPS Simplified Acute Physiology Score, VAP ventilator-associated pneumonia, MV mechanical ventilation, HR hazard ratio, S. maltophilia Stenotrophomonas maltophilia, SOFA Sequential Organ Failure Assessment

in the multivariate analysis because it was a consequence and not present at the diagnosis of $S$. maltophilia. The results were similar when only considering VAP in our cohort $(N=228)$ (Additional file 5: Table S4). Finally, we performed a statistical analysis based on a propensity score to evaluate the effect of an empirical antibiotic therapy effective on S. maltophilia on the primary endpoint (Additional file 6: Table S5). After matching, we compared 48 patients who received appropriate empirical antibiotic therapy versus 222 who did not (Additional file 7: Table S6). This analysis confirmed the previous results $(\mathrm{HR}=$ 0.891, 95\%CI [0.498-1.593], $p=0.697$ ).

\section{Mono- and polymicrobial S. maltophilia HAP}

The aforementioned results remained unchanged when considering only monomicrobial S. maltophilia pneumonia $(n=117)(\mathrm{HR}=1.08,95 \% \mathrm{CI}[1.01 ; 1.15]$, $p=0.021$ for SOFA score at $S$. maltophilia pneumonia diagnosis). Comparisons of characteristics and outcomes between mono- and polymicrobial S. maltophilia HAP are provided in Table 5. No differences were noted in in-hospital death irrespective of an appropriate and timely empiric antimicrobial therapy between mono- versus polymicrobial S. maltophilia HAP. A similar number of VAP occurred in both groups, 91 $(77.8 \%)$ versus $136(82.9 \%)(p=0.280)$ for mono- and polymicrobial HAP respectively. The duration of ventilation prior to $S$. maltophilia HAP diagnosis and ICU length of stay were shorter in patients with monomicrobial S. maltophilia HAP.

\section{Discussion}

Herein, we report the largest cohort study of critically ill patients developing S. maltophilia HAP. Regarding the large screening, the prevalence of $S$. maltophilia HAP remained very low. The majority of $S$. maltophilia HAP was VAP and occurred in patients ventilated 
Table 5 Characteristics and outcomes comparing patients with mono- versus polymicrobial Stenotrophomonas maltophilia HAPs

\begin{tabular}{|c|c|c|c|}
\hline Variables & Sm only $N=117$ & Polymicrobial Sm HAP N = 164 & $p$ value \\
\hline Gender, male & $78(66.7)$ & $119(72.6)$ & 0.362 \\
\hline Age, years & $67[59-76]$ & $64[55-72]$ & 0.024 \\
\hline BMI $\left(\mathrm{kg} \mathrm{m}^{-2}\right)$ & $24.6[21.1-29.6]$ & $25.7[22.3-28.4]$ & 0.361 \\
\hline Reason for ICU admission & & & 0.024 \\
\hline Medical condition & $80(68.4)$ & $87(53)$ & \\
\hline Scheduled surgery & $8(6.8)$ & $24(14.6)$ & \\
\hline Emergent surgery & $29(24.8)$ & $53(32.3)$ & \\
\hline Previous carriage of S. maltophilia & $5(4.3)$ & $6(3.7)$ & 0.812 \\
\hline \multicolumn{4}{|l|}{ Pulmonary comorbidities } \\
\hline Chronic obstructive pulmonary disease & $27(23.1)$ & $24(14.6)$ & 0.084 \\
\hline Chronic respiratory insufficiency & $15(12.8)$ & $14(8.5)$ & 0.245 \\
\hline Cystic fibrosis & $0(0)$ & $2(1.2)$ & 0.512 \\
\hline Prior exposure to carbapenems & $27(23.1)$ & $37(22.5)$ & 0.723 \\
\hline Susceptibility to trimethoprim-sulfamethoxazole & $97(82.9)$ & $147(89.6)$ & 0.424 \\
\hline \multicolumn{4}{|l|}{ Severity scores } \\
\hline SOFA score at admission & $8[5-11]$ & $8[5-11]$ & 0.381 \\
\hline SAPS $\|$ at $24 \mathrm{~h}$ & $48[37-62]$ & $47[36-63]$ & 0.672 \\
\hline Ventilator-associated pneumonia & $91(77.8)$ & $136(82.9)$ & 0.280 \\
\hline Duration of mechanical ventilation prior to S. maltophilia HAP, days & $9[2-17]$ & $11[6-20]$ & 0.031 \\
\hline ICU length of stay, days & $28[16-49]$ & $36[22-60]$ & 0.034 \\
\hline Hospital length of stay, days & $45[26-80]$ & 59 [32-98] & 0.062 \\
\hline Number of days between hospital admission and S. maltophilia HAP & 14 [8-23] & $17[8-31]$ & 0.294 \\
\hline Number of days between ICU admission and S. maltophilia HAP & $10[5-18]$ & $12[6-20]$ & 0.046 \\
\hline Empiric antibiotic therapy & $67(57.3)$ & $99(60.4)$ & 0.602 \\
\hline Combination therapy active on S. maltophilia & $66(56.4)$ & $100(61.0)$ & 0.407 \\
\hline Overall in-hospital mortality & $63(53.8)$ & $74(45.1)$ & 0.228 \\
\hline In-hospital mortality according to empiric antimicrobial therapy & & & 0.273 \\
\hline Appropriate & $8(6.8)$ & $16(9.8)$ & \\
\hline Inappropriate & $24(20.5)$ & $30(18.3)$ & \\
\hline
\end{tabular}

Data are presented as median, interquartile range ([IQR]), or number (percentage) $(n,(\%))$ as appropriate

BMI body mass index, HAP hospital-acquired pneumonia, SAPS Simplified Acute Physiology Score, SOFA Sequential Organ Failure Assessment, S. maltophilia Stenotrophomonas maltophilia

for more than 10 days and previously exposed to several antimicrobial therapies. The mortality rate of these patients remained high, but surprisingly, the treatment delay in adequate antimicrobial therapy targeting S. maltophilia was not found to be associated with mortality. This observation may be the result of (i) a low virulence of the pathogen, (ii) the underlying condition of the critically ill patient being more contributive to the outcome than $S$. maltophilia HAP itself, or (iii) a 24- to 48-h delay in the treatment of $S$. maltophilia HAP had no real impact. Finally, if SAPSII and SOFA score were independently associated with mortality, no specific pneumonia or antimicrobial therapy-related factors impacted the outcome.
Our study population shares common features with previously published reports [3, 4, 6, 7, 19-23]. Indeed, S. maltophilia pneumonia develops in high-risk phenotypes patients, i.e., long ICU/hospital length of stay and duration of mechanical ventilation. Despite a mortality rate of approximately $50 \%$, it is difficult to delineate direct attributable mortality of $S$. maltophilia HAP from mortality linked to underlying diseases [24]. Indeed, the prolonged ICU length of stay preceding S. maltophilia isolation and the number of prior antimicrobial therapies suggest noticeable patient frailty and complicated medical history. These factors have been associated to high mortality in patients with resistant bacteria in ICU [25]. S. maltophilia HAP could also be perceived as a final 
septic insult in long-stayer patients, promoting care withdrawal from the medical team.

Previous studies that included a small number of patients $[3,4,6,19-23,26-31]$ suggested that immunecompromised conditions, COPD, prior cardiac surgery, or prior antimicrobial therapy were risk factors for $S$. maltophilia HAP. Conversely, our large series of mixed ICU patients did not confirm these elements. This implies that the involvement of $S$. maltophilia in late onset HAP should be considered and be kept in mind by all critical care physicians. However, in our series, initial antimicrobial therapy inactive against $S$. maltophilia was not a risk factor for in-hospital mortality, arguing against a systematic coverage of $S$. maltophilia by empirical antimicrobial therapy in this setting.

Although the prolonged duration of antimicrobial treatment is a well-known risk factor for emergence of multidrug resistant (MDR) bacteria [32], it did not appear to be discriminant in our study, irrespective of the class of antimicrobial agent previously administered. These results are in accordance with previously published literature on continuation or de-escalation of beta-lactam antibiotics and emergence of MDR [31]. Indeed, different regimens were used in our population, with various durations of treatment before S. maltophilia HAP diagnosis without apparent consequences on $S$. maltophilia emergence and susceptibility profiles. Soubirou et al. found that the increase in use of antimicrobial class was an independent predictor of S. maltophilia emergence in VAP [33]. It is however conceivable that some patients of our cohort may be colonized with other non-fermenting Gram-negative bacilli. Actually, almost $20 \%$ of patients had COPD or chronic respiratory insufficiency and might be regularly exposed to antibiotics. Nseir et al. and Saugel et al. reported 63\% and 25\% respectively incidence of COPD patients with S. maltophilia pneumonia [3, 4].

Despite clinical signs of HAP, only $59 \%$ of patients readily received empirical antimicrobial therapy (Additional file 4: Table S3). This highlights the variable implementation and adherence to antimicrobial bundles of care and stewardship programs [34-36]. Pathmanathan et al. previously reported no measurable impact of antibiotic therapy in patients without evidence of consolidation which suggests colonization [23]. In our study, colonization was excluded. Although it is currently suggested to start antibiotics early in patients with suspected VAP [37], physicians may have been expecting a definitive identification with the resistance profile of microorganisms possibly involved to restrain the use of broad-spectrum antibiotics, especially in patients previously exposed to several antibiotic regimens. Tracheobronchitis and pneumonia may also be hard to be differentiated and need time to be distinguished.
Of note, the interplay between resistance and virulence remains complex [38]. In these patients already exposed to several series of antimicrobial therapies, with an extended hospital length of stay, the likelihood of MDR bacteria involvement was very high. Moreover, due to its natural resistance to multiple antimicrobial agents, only one third of empirical antimicrobial therapies was actually effective against $S$. maltophilia. Conversely to other authors [39], but in accordance with studies on Pseudomonas aeruginosa VAP, the delayed administration of effective antimicrobial treatment was not statistically associated with increased mortality [40, 41].

The duration of $S$. maltophilia HAP antimicrobial therapy is still subject to debate. The comparison of short ( $<8$ days) versus prolonged ( 8 days and greater) antibiotic course could not properly be investigated in our study because of its design. Chastre et al. demonstrated that an 8-day course of antibiotic therapy for VAP was not inferior to a longer duration, but only $0.8 \%$ of patients had an S. maltophilia VAP [42]. However, it was suggested that patients infected with difficult-to-treat pathogens, immunocompromised patients, and patients at high risk for relapse may require a longer duration of antibiotic therapy. In our study, we identified neither the duration of antimicrobial treatment nor the combination of antibiotic therapies as significant risk factors for in-hospital death. Low virulence of $S$. maltophilia strains may partially explain these findings. In a recent retrospective study focused on the interest of combination therapy, Shah et al. reported that combination of antibiotic therapies yielded similar clinical efficacy and resistance development compared to monotherapy [43].

Optimal antimicrobial against S. maltophilia HAP may raise some concerns. The $S$. maltophilia strains in our study had a preserved susceptibility to ticarcillin-clavulanate and TMP-SMX, 73 and $88 \%$ respectively as expected [44]. However, only $29 \%$ of S. maltophilia HAP were treated with TMP-SMX. These discrepancies may be related to ICU physicians' habits, the fear of TMPSMX-related side effects, and the type of antibiotics administered prior to occurrence of S. maltophilia HAP. The use of fluoroquinolones could have been considered easier. When prescribed, TMP-SMX was combined with another antibiotic effective on S. maltophilia in $80 \%$ of cases. In addition, antimicrobial agent shortages change antimicrobial therapy armamentarium, with a cessation of manufacture of ticarcillin-clavulanate in 2015 [45]. Although ceftazidime/avibactam has poor activity on $S$. maltophilia [46], it may restore the susceptibility to aztreonam through the inhibition of the L2 $\beta$-lactamase in vitro [47]. The clinical efficacy of this combination has been reported in a case report of a transplant renal patient [48]. 
Augmented renal clearance can alter the pharmacokinetics and pharmacodynamics (PK/PD) of several antimicrobial agents, mainly ß-lactams [49]. The detailed dosages of antimicrobial agents and the measured creatinine clearance were not collected in the present study. However, TMP-SMX and fluoroquinolones were the most prescribed agents. Their plasma concentrations are not dramatically influenced by augmented renal clearance and not easily monitored in daily practice.

\section{Limitations}

Our study differs from previous studies, where patients with and without $S$. maltophilia infection were compared. We did not consider S. maltophilia colonizations but only HAP, unlike previous studies [4]. In the case of a polymicrobial sample, it is uncertain which bacteria were responsible for the HAP. One may argue that our study suffers from inaccurate diagnoses differentiating between VAP and ventilator-associated tracheobronchitis due to its retrospective design using ICD codes. We acknowledge that the diagnosis method (ETA versus BAL or PSB) may influence the detection of S. maltophilia. This was a pragmatic study that describes different ICU practices, and to date, there is no formal evidence of improved outcomes depending on the diagnosis method used [50]. Despite strict inclusion criteria, and search for consensus in case of debatable case, it is possible that the physician's judgment and diagnosis reported in the medical record were inaccurate. However, $80 \%$ of HAP were VAP in our study and excluding nonventilated patients did not alter the observed results.

\section{Conclusions}

S. maltophilia HAP had a very low incidence in critically ill patients but was associated with high mortality rate in this large multicenter study. Its onset is hard to predict because of lack of specific risk factors but occurs mainly in long-stay ICU patients. The present study did not provide evidence of a significant effect of delay, duration, or combination of antimicrobial therapy on mortality. Efforts in developing novel and effective approaches for prevention are warranted.

\section{Supplementary information}

Supplementary information accompanies this paper at https://doi.org/10. 1186/s13054-019-2649-5.

Additional file 1. List of participating centers, collaborators and case-mix of ICU patients.

Additional file 2: Table S1. Invasive devices inserted at the diagnosis of Stenotrophomonas maltophilia hospital-acquired pneumonia.

Description of invasive devices inserted at the diagnosis of Stenotrophomonas maltophilia hospital-acquired pneumonia.
Additional file 3: Table S2. Diagnosis methods for isolation of Stenotrophomonas maltophilia. Description of diagnosis methods for isolation of Stenotrophomonas maltophilia.

Additional file 4: Table S3. Treatment failure of Stenotrophomonas maltophilia hospital-acquired pneumonia. Description of treatment failures of Stenotrophomonas maltophilia hospital-acquired pneumonia.

Additional file 5: Table S4. Variables associated with the time to inhospital death in patients with $S$. maltophilia ventilator-associated pneumonia. Variables associated with the time to in-hospital death in patients with S. maltophilia ventilator-associated pneumonia.

Additional file 6: Table S5. Propensity Score Matching. Time to inhospital death was compared between matched groups using a Cox proportional hazard model.

Additional file 7: Table S6. Variable associated with time-to-death in the propensity matched population. Variable associated with timeto-death in the propensity matched population.

\section{Abbreviations}

ARDS: Acute respiratory distress syndrome; AST: Antimicrobial susceptibility testing; BAL: Bronchoalveolar lavage; CFU: Colony-forming units; ETA: Endotracheal aspirate; HAP: Hospital-acquired pneumonia; ICU: Intensive care unit; MDR: Multidrug resistant; PSB: Protected specimen brush; PTC: Protected (plugged) telescoping catheter; SAPS: Simplified Acute Physiology Score; SOFA: Sequential organ failure assessment; TMPSMX: Trimethoprim-sulfamethoxazole; VAP: Ventilator-associated pneumonia

\section{Acknowledgements}

The authors would like to thank Geoffroy Cagninacci and Cédric Baumann for their excellent implementation and support of RedCap.

Prof Jean-Michel Constantin MD. PhD: Pôle de Médecine Péri-Opératoire (MPO), Centre Hospitalier Universitaire de Clermont-Ferrand, ClermontFerrand, France ; mixed ICU.

Dr Thomas Godet MD.: Réanimation Adultes et Soins Continus, Pôle de Médecine Péri-opératoire, Hôpital Estaing, CHU de Clermont-Ferrand, France ; mixed ICU.

Dr Philippe Guerci MD.: Department of Anesthesiology and Critical Care Medicine, Institut Lorrain du Coeur et des Vaisseaux, University Hospital of Nancy, Vandoeuvre-Lès-Nancy, France; mixed surgical ICU.

Dr Sebastien Perbet MD. PhD.: Réanimation Médico-Chirurgicale, Pôle de Médecine Péri-opératoire, Hôpital Gabriel Montpied, CHU de ClermontFerrand, France ; mixed ICU.

Dr Stanislas Ledochowski MD.: Service de Réanimation Polyvalente, Groupement Hospitalier Nord Dauphiné- Centre Hospitalier Pierre Oudot, Bourgoin-Jallieu, France; mixed ICU.

\section{Take-home message}

In critically ill patients with Stenotrophomonas maltophilia hospital-acquired pneumonia, delay to appropriate antimicrobial treatment, combination antimicrobial therapy, or prolonged antimicrobial therapy may not be associated with increased survival.

\section{Authors' contributions}

$P G, H B$, and $A B$ contributed to the study design, screening for eligibility and inclusion of patients, data analysis, writing of the first draft and of the final version, and review of the manuscript. NM contributed to the screening for eligibility and inclusion of patients, data analysis, writing of the first draft and of the final version, and review of the manuscript. DH contributed to the writing of the first draft and of the final version, data analysis, and review of the manuscript. SL and EN contributed to the screening for eligibility and inclusion of patients, writing of the last draft, and review of the manuscript. $M M, J G, C C, G L, P T, C D, A K, T G, J P$, and J-ML contributed to the screening for eligibility and inclusion of patients and review of the manuscript. All authors read and approved the final manuscript.

\section{Funding}

This study has been solely funded by the Department of Anaesthesiology and Critical Care Medicine of the University Hospital of Nancy, France. The Unit of Methodology, Data and Statistics kindly provided free of charge RedCap support. 


\section{Availability of data and materials}

The datasets analyzed in this study are not publicly available due to privacy issues, but are available from the corresponding author upon reasonable request.

\section{Ethics approval and consent to participate}

This study was approved by the ethics committee of the Sociéte Française d'Anesthésie et de Réanimation (IRB 00010254-2015-010; February 19, 2016). This study was the subject of a favorable decision from the Comité consultatif sur le traitement de l'information en matière de recherche dans le domaine de la santé on April 20, 2017, and of a declaration to the Comission Nationale Informatique et Libertés on May 20, 2017.

This study complies with the principles of the 1964 Declaration of Helsinki in reviewing and publishing information from the patient's medical records. The requirement for informed consent from each patient was waived because the design of study was retrospective in nature and because of the use of anonymized patient and hospital data.

\section{Consent for publication}

All the authors have read the manuscript, approved this submission, and consented for publication.

\section{Competing interests}

The authors declare that they have no competing interests.

\section{Author details}

${ }^{1}$ Department of Anaesthesiology and Critical Care Medicine, Institut Lorrain du Coeur et des Vaisseaux, University Hospital of Nancy-Brabois, Vandoeuvre-Lès-Nancy, France. ${ }^{2}$ INSERM U1116, Groupe Choc, University of Lorraine, Nancy, France. ${ }^{3}$ Sorbonne Université, Assistance Publique - Hôpitaux de Paris (AP-HP), Department of Anaesthesiology and Critical Care Medicine, Institute of Cardiology, Pitié-Salpêtrière Hospital, 47-83 Boulevard de I'Hôpital, 75013 Paris, France. ${ }^{4}$ Service d'Anesthésie-Réanimation Chirurgicale, Hôpital Hautepierre, Hôpitaux Universitaires de Strasbourg, Strasbourg, France. ${ }^{5}$ Service d'Anesthésie-Réanimation, Hôpital Henri Mondor, DMU CARE, Assistance Publique - Hôpitaux de Paris (AP-HP), Inserm U955 équipe 3, Université Paris-Est Créteil, Créteil, France. ${ }^{6}$ Réanimation Chirurgicale Polyvalente, Hôpital Central, Centre Hospitalier Universitaire de Nancy, Nancy, France. 'Réanimation polyvalente, Hôpital de Mercy, CHR Metz-Thionville, Metz, France. ${ }^{8}$ Service d'Anesthésie-Réanimation, Hôpital Bichat-Claude Bernard, Assistance Publique - Hôpitaux de Paris (AP-HP), Paris, France. ${ }^{9}$ Réanimation polyvalente, Hôpital d'Instruction des Armées (HIA) Bégin, Saint-Mandé, France. ${ }^{10}$ Service de Réanimation Polyvalente, Groupement Hospitalier Nord Dauphiné- Centre Hospitalier Pierre Oudot, Bourgoin-Jallieu, France. ${ }^{11}$ Réanimation Médicale, Institut Lorrain du Cœur et des Vaisseaux, CHU Nancy-Brabois, Vandoeuvre-Lès-Nancy, France. ${ }^{12}$ Réanimation Adultes et Soins Continus, Pôle de Médecine Péri-opératoire, Hôpital Estaing, Centre Hospitalier Universitaire de Clermont-Ferrand, Clermont-Ferrand, France.

${ }^{13}$ Faculté de Médecine, Institut de Physiologie, EA3072, Fédération de Médecine Translationnelle de Strasbourg (FMTS), Université de Strasbourg, Strasbourg, France. ${ }^{14}$ Service d'Anesthésie-Réanimation, Réanimation polyvalente, Centre Hospitalier Emile Durkheim, Epinal, France.

${ }^{15}$ Département Biostatistique Santé Publique Et Information Médicale, Unité de Recherche Clinique PSL-CFX, Centre de Pharmacoépidémiologie (Cephepi), Sorbonne Université, INSERM, Institut Pierre Louis de Santé Publique, Equipe Pharmacoépidémiologie et évaluation des soins, AP-HP, Hôpital Pitié-Salpêtrière, CIC-1421, Paris, France.

\section{Received: 4 June 2019 Accepted: 15 October 2019}

\section{Published online: 21 November 2019}

\section{References}

1. Magill SS, Edwards JR, Bamberg W, Beldavs ZG, Dumyati G, Kainer MA, et al. Multistate point-prevalence survey of health care-associated infections. N Engl J Med. 2014;370:1198-208.

2. Healthcare-associated infections in intensive care units - Annual Epidemiological Report for 2015. European Centre for Disease Prevention and Control. 2017. Available from: http://ecdc.europa.eu/en/publicationsdata/healthcare-associated-infections-intensive-care-units-annualepidemiological. [cited 2018 Aug 27]
3. Nseir S, Di Pompeo C, Brisson H, Dewavrin F, Tissier S, Diarra M, et al. Intensive care unit-acquired Stenotrophomonas maltophilia: incidence, risk factors, and outcome. Crit Care. 2006;10:R143.

4. Saugel B, Eschermann K, Hoffmann R, Hapfelmeier A, Schultheiss C, Phillip V, et al. Stenotrophomonas maltophilia in the respiratory tract of medical intensive care unit patients. Eur J Clin Microbiol Infect Dis. 2012;31:1419-28.

5. Metan G, Hayran M, Hascelik G, Uzun O. Which patient is a candidate for empirical therapy against Stenotrophomonas maltophilia bacteraemia? An analysis of associated risk factors in a tertiary care hospital. Scand J Infect Dis. 2006;38:527-31.

6. Hanes SD, Demirkan K, Tolley E, Boucher BA, Croce MA, Wood GC, et al. Risk factors for late-onset nosocomial pneumonia caused by Stenotrophomonas maltophilia in critically ill trauma patients. Clin Infect Dis. 2002;35:228-35.

7. Falagas ME, Kastoris AC, Vouloumanou EK, Rafailidis PI, Kapaskelis AM, Dimopoulos G. Attributable mortality of Stenotrophomonas maltophilia infections: a systematic review of the literature. Future Microbiol. 2009;4 1103-9.

8. Leone M, Constantin J-M, Dahyot-Fizelier C, Duracher-Gout C, JoannesBoyau $\mathrm{O}$, Langeron $\mathrm{O}$, et al. French intensive care unit organisation. Anaesth Crit Care Pain Med. 2018;37:625-7.

9. Torres A, Niederman MS, Chastre J, Ewig S, Fernandez-Vandellos P, Hanberger $\mathrm{H}$, et al. International ERS/ESICM/ESCMID/ALAT guidelines for the management of hospital-acquired pneumonia and ventilator-associated pneumonia: guidelines for the management of hospital-acquired pneumonia (HAP)/ventilator-associated pneumonia (VAP) of the European Respiratory Society (ERS), European Society of Intensive Care Medicine (ESICM), European Society of Clinical Microbiology and Infectious Diseases (ESCMID) and Asociación Latinoamericana del Tórax (ALAT). Eur Respir J. 2017;50:1700582.

10. Raoof S, Baumann MH, Critical Care Societies Collaborative. An official multisociety statement: ventilator-associated events: the new definition Crit Care Med 2014;42:228-229.

11. Acute Respiratory Distress Syndrome. The Berlin definition. JAMA. 2012;307: 2526-33.

12. Kalil AC, Metersky ML, Klompas M, Muscedere J, Sweeney DA, Palmer LB, et al. Management of adults with hospital-acquired and ventilatorassociated pneumonia: 2016 clinical practice guidelines by the Infectious Diseases Society of America and the American Thoracic Society. Clin Infect Dis. 2016;63:e61-111.

13. Matuschek E, Brown DFJ, Kahlmeter G. Development of the EUCAST disk diffusion antimicrobial susceptibility testing method and its implementation in routine microbiology laboratories. Clin Microbiol Infect. 2014;20:255-66.

14. Harris PA, Taylor R, Thielke R, Payne J, Gonzalez N, Conde JG. Research electronic data capture (REDCap)--a metadata-driven methodology and workflow process for providing translational research informatics support. J Biomed Inform. 2009:42:377-81.

15. Toulouse E, Masseguin C, Lafont B, McGurk G, Harbonn A, Roberts AJ, et al. French legal approach to clinical research. Anaesth Crit Care Pain Med. 2018;37:607-14.

16. Austin PC. Optimal caliper widths for propensity-score matching when estimating differences in means and differences in proportions in observational studies. Pharm Stat. 2011;10:150-61.

17. Austin PC. An introduction to propensity score methods for reducing the effects of confounding in observational studies. Multivariate Behav Res. 2011;46:399-424.

18. Resche-Rigon M, Pirracchio R, Robin M, De Latour RP, Sibon D, Ades L, et al. Estimating the treatment effect from non-randomized studies: the example of reduced intensity conditioning allogeneic stem cell transplantation in hematological diseases. BMC Blood Disord. 2012;12:10.

19. Martin-Loeches I, Deja M, Koulenti D, Dimopoulos G, Marsh B, Torres A, et al. Potentially resistant microorganisms in intubated patients with hospitalacquired pneumonia: the interaction of ecology, shock and risk factors. Intensive Care Med. 2013;39:672-81.

20. Gopalakrishnan R, Hawley HB, Czachor JS, Markert RJ, Bernstein JM. Stenotrophomonas maltophilia infection and colonization in the intensive care units of two community hospitals: a study of 143 patients. Heart Lung. 1999;28:134-41.

21. Villarino ME, Stevens LE, Schable B, Mayers G, Miller JM, Burke JP, et al. Risk factors for epidemic Xanthomonas maltophilia infection/colonization in intensive care unit patients. Infect Control Hosp Epidemiol. 1992;13:201-6. 
22. Maningo E, Watanakunakorn C. Xanthomonas maltophilia and Pseudomonas cepacia in lower respiratory tracts of patients in critical care units. J Inf Secur. 1995;31:89-92.

23. Pathmanathan A, Waterer GW. Significance of positive Stenotrophomonas maltophilia culture in acute respiratory tract infection. Eur Respir J. 2005;25 911-4.

24. Bekaert M, Timsit J-F, Vansteelandt S, Depuydt P, Vésin A, Garrouste-Orgeas M, et al. Attributable mortality of ventilator-associated pneumonia: a reappraisal using causal analysis. Am J Respir Crit Care Med. 2011;184:1133-9.

25. Barbier F, Lisboa T, Nseir S. Understanding why resistant bacteria are associated with higher mortality in ICU patients. Intensive Care Med. 2016; 42:2066-9.

26. Lee M-R, Wang H-C, Yang C-Y, Lin C-K, Kuo H-Y, Ko J-C, et al. Clinical characteristics and outcomes of patients with pleural infections due to Stenotrophomonas maltophilia at a medical center in Taiwan, 2004-2012. Eur J Clin Microbiol Infect Dis. 2014;33:1143-8.

27. Garcia Paez Jl, Tengan FM, Barone AA, Levin AS, Costa SF. Factors associated with mortality in patients with bloodstream infection and pneumonia due to Stenotrophomonas maltophilia. Eur J Clin Microbiol Infect Dis. 2008;27:901-6.

28. Chawla K, Vishwanath S, Gupta A. Stenotrophomonas maltophilia in lower respiratory tract infections. J Clin Diagn Res. 2014;8:DC20-2.

29. Batra P, Mathur P, Misra MC. Clinical characteristics and prognostic factors of patients with Stenotrophomonas maltophilia infections. J Lab Physicians. 2017:9:132-5.

30. Fujita J, Yamadori I, Xu G, Hojo S, Negayama K, Miyawaki H, et al. Clinical features of Stenotrophomonas maltophilia pneumonia in immunocompromised patients. Respir Med. 1996;90:35-8.

31. De Bus L, Denys W, Catteeuw J, Gadeyne B, Vermeulen K, Boelens J, et al. Impact of de-escalation of beta-lactam antibiotics on the emergence of antibiotic resistance in ICU patients: a retrospective observational study. Intensive Care Med. 2016;42:1029-39.

32. Kollef MH. Is antibiotic cycling the answer to preventing the emergence of bacterial resistance in the intensive care unit? Clin Infect Dis. 2006;43:S82-8.

33. Soubirou J-F, Gault N, Alfaiate T, Lolom I, Tubach F, Andremont A, et al. Ventilator-associated pneumonia due to carbapenem-resistant Gramnegative bacilli in an intensive care unit without carbapenemase-producing Enterobacteriaceae or epidemic Acinetobacter baumannii. Scand J Infect Dis. 2014;46:215-20.

34. Bouadma L, Deslandes E, Lolom I, Le Corre B, Mourvillier B, Regnier B, et al. Long-term impact of a multifaceted prevention program on ventilatorassociated pneumonia in a medical intensive care unit. Clin Infect Dis. 2010; 51:1115-22

35. Álvarez-Lerma F, Grau S, Echeverría-Esnal D, Martínez-Alonso M, GraciaArnillas MP, Horcajada JP, et al. A before-and-after study of the effectiveness of an antimicrobial stewardship program in critical care. Antimicrob Agents Chemother. 2018;62:e01825-17.

36. Lindsay PJ, Rohailla S, Taggart LR, Lightfoot D, Havey T, Daneman N, et al. Antimicrobial stewardship and intensive care unit mortality: a systematic review. Clin Infect Dis. 2019;68:748-56.

37. Luyt C-E, Bréchot N, Trouillet J-L, Chastre J. Antibiotic stewardship in the intensive care unit. Crit Care. 2014;18:480.

38. Beceiro A, Tomás M, Bou G. Antimicrobial resistance and virulence: a successful or deleterious association in the bacterial world? Clin Microbiol Rev. 2013;26:185-230.

39. Iregui M, Ward S, Sherman G, Fraser VJ, Kollef MH. Clinical importance of delays in the initiation of appropriate antibiotic treatment for ventilatorassociated pneumonia. Chest. 2002;122:262-8.

40. Planquette B, Timsit J-F, Misset BY, Schwebel C, Azoulay E, Adrie C, et al. Pseudomonas aeruginosa ventilator-associated pneumonia. Predictive factors of treatment failure. Am J Respir Crit Care Med. 2013;188:69-76.

41. Peña C, Gómez-Zorrilla S, Oriol I, Tubau F, Dominguez MA, Pujol M, et al. Impact of multidrug resistance on Pseudomonas aeruginosa ventilatorassociated pneumonia outcome: predictors of early and crude mortality. Eur J Clin Microbiol Infect Dis. 2013;32:413-20

42. Chastre J, Wolff M, Fagon J-Y, Chevret S, Thomas F, Wermert D, et al. Comparison of 8 vs 15 days of antibiotic therapy for ventilator-associated pneumonia in adults: a randomized trial. JAMA. 2003;290:2588-98.

43. Shah MD, Coe KE, El Boghdadly Z, Wardlow LC, Dela-Pena JC, Stevenson KB, et al. Efficacy of combination therapy versus monotherapy in the treatment of Stenotrophomonas maltophilia pneumonia. J Antimicrob Chemother. 2019;74:2055-9.
44. Sader HS, Farrell DJ, Flamm RK, Jones RN. Antimicrobial susceptibility of Gram-negative organisms isolated from patients hospitalised with pneumonia in US and European hospitals: results from the SENTRY Antimicrobial Surveillance Program, 2009-2012. Int J Antimicrob Agents. 2014;43:328-34

45. Tsiodras S, Pittet D, Carmeli Y, Eliopoulos G, Boucher H, Harbarth S. Clinical implications of Stenotrophomonas maltophilia resistant to trimethoprimsulfamethoxazole: a study of 69 patients at 2 university hospitals. Scand J Infect Dis. 2000;32:651-6.

46. Hachem R, Reitzel R, Rolston K, Chaftari AM, Raad I. Antimicrobial Activities of Ceftazidime-Avibactam and Comparator Agents against clinical bacteria isolated from patients with cancer. Antimicrob Agents Chemother. 2017; 61(4).

47. Mojica MF, Papp-Wallace KM, Taracila MA, Barnes MD, Rutter JD, Jacobs MR, et al. Avibactam restores the susceptibility of clinical isolates of Stenotrophomonas maltophilia to aztreonam. Antimicrob Agents Chemother. 2017;61(10)

48. Mojica MF, Ouellette CP, Leber A, Becknell MB, Ardura MI, Perez F, et al. Successful treatment of bloodstream infection due to metallo- $\beta$-lactamaseproducing Stenotrophomonas maltophilia in a renal transplant patient. Antimicrob Agents Chemother. 2016;60:5130-4.

49. Veiga RP, Paiva J-A. Pharmacokinetics-pharmacodynamics issues relevant for the clinical use of beta-lactam antibiotics in critically ill patients. Crit Care. 2018;22:233.

50. Berton DC, Kalil AC, Teixeira PJ. Quantitative versus qualitative cultures of respiratory secretions for clinical outcomes in patients with ventilatorassociated pneumonia. Cochrane Database Syst Rev. 2014;(10):CD006482.

\section{Publisher's Note}

Springer Nature remains neutral with regard to jurisdictional claims in published maps and institutional affiliations.
Ready to submit your research? Choose BMC and benefit from:

- fast, convenient online submission

- thorough peer review by experienced researchers in your field

- rapid publication on acceptance

- support for research data, including large and complex data types

- gold Open Access which fosters wider collaboration and increased citations

- maximum visibility for your research: over $100 \mathrm{M}$ website views per year

At BMC, research is always in progress.

Learn more biomedcentral.com/submissions 